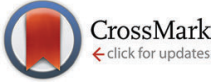

Cite this: J. Mater. Chem. B, 2016, 4, 3135

Received 1st February 2016 Accepted 30th March 2016

DOI: $10.1039 / c 6 t b 00281 a$

www.rsc.org/MaterialsB

\title{
Silica core-shell particles for the dual delivery of gentamicin and rifamycin antibiotics $\dagger$
}

\author{
Andrea M. Mebert, ${ }^{\text {ab }}$ Carole Aimé, ${ }^{c}$ Gisela S. Alvarez, ${ }^{a b}$ Yupeng Shi, ${ }^{c}$ Sabrina A. Flor, ${ }^{d}$ \\ Silvia E. Lucangioli, ${ }^{d}$ Martin F. Desimone ${ }^{\star a b}$ and Thibaud Coradin ${ }^{\star c}$
}

\begin{abstract}
Increasing bacterial resistance calls for the simultaneous delivery of multiple antibiotics. One strategy is to design a unique pharmaceutical carrier that is able to incorporate several drugs with different physicochemical properties. This is highly challenging as it may require the development of compartmentalization approaches. Here we have prepared core-shell silica particles allowing for the dual delivery of gentamicin and rifamycin. The effect of silica particle surface functionalization on antibiotic sorption was first studied, enlightening the role of electrostatic and hydrophobic interactions. This in turn dictates the chemical conditions for shell deposition and further sorption of these antibiotics. In particular, the silica shell deposition was favored by the positively charged layer of gentamicin coating on the core particle surface. Shell modification by thiol groups finally allowed for rifamycin sorption. The antibacterial activity of the core-shell particles against Staphylococcus aureus and Pseudomonas aeruginosa demonstrated the dual release and action of the two antibiotics.
\end{abstract}

\section{Introduction}

Drug carriers for local antibiotic delivery have gained attention in the last few years due to the need to deliver effective antibiotic doses, and avoid the high toxicity associated with systemic administration. ${ }^{1}$ Those delivery systems can be applied in situations such as periodontal disease ${ }^{2}$ orthopedic trauma ${ }^{3-5}$ or in skin wound healing. ${ }^{6}$ However, due to the presence of multiple pathogens, and the increasing drug resistance, the co-delivery of different antibiotics or other compounds is a promising alternative for increasing treatment efficacy. ${ }^{7}$

When the antimicrobial treatment is associated with the implantation of a biomaterial, the relevant drugs are ideally loaded within the biomaterial scaffold or at its surface. ${ }^{8}$ However, for soft tissue repair where hydrogel-based dressings are used, the kinetics of drug release is usually too fast due to the large porosity of the scaffold compared to antibiotic dimensions. ${ }^{9}$

\footnotetext{
${ }^{a}$ Universidad de Buenos Aires, Facultad de Farmacia y Bioquimica, Junin 956 Piso 3, (1113) Ciudad Autónoma de Buenos Aires, Argentina. E-mail: desimone@ffyb.uba.ar

${ }^{b}$ IQUIMEFA-CONICET, Ciudad Autónoma de Buenos Aires, Argentina

${ }^{c}$ Sorbonne Universités, UPMC Univ Paris 06, CNRS, Collège de France, UMR 7574, Laboratoire de Chimie de la Matière Condensée de Paris,

11 Place Marcelin Berthelot, F-75005 Paris, France

${ }^{d}$ CONICET - Universidad de Buenos Aires, Facultad de Farmacia y Bioquímica, Departamento de Tecnología Farmacéutica, Ciudad Autónoma de Buenos Aires, Argentina

$\dagger$ Electronic supplementary information (ESI) available: Additional TEM and SEM images of nanoparticles and MS data of antibiotics. See DOI: 10.1039/c6tb00281a
}

Another strategy is to introduce strong chemical bonds between the material and the drug but this can become complex if multiple drug delivery is targeted.

As an alternative, the use of nanocomposite materials where the drugs are associated with nanoparticles embedded in the scaffold has attracted increasing attention. ${ }^{10,11}$ The intrinsic properties of these nanoparticles, as well as their interactions with the host hydrogel, can be tuned so as to optimize the drug release profiles. Among possible antibiotic carriers, silica nanoparticles have been extensively studied. They can be prepared in a wide range of size, porosity and chemical composition. Whereas a full assessment of their biocompatibility is still to be achieved, ${ }^{12}$ numerous studies have demonstrated that silica nanoparticles with size ranging from $10 \mathrm{~nm}$ to $200 \mathrm{~nm}$ exhibit limited cytotoxicity. ${ }^{13-16}$ Moreover, grafting sulfonate, amine and especially thiol moieties further decreases their detrimental effect. ${ }^{17}$

To address the issues related to co-delivery of antibiotics, two options can be envisioned. The first one would rely on the incorporation of different populations of particles, each loaded with a unique drug. Although each drug-carrier can be individually optimized, this approach requires the use of a high total concentration of silica particles. Alternatively, the incorporation of multiple drugs within a single type of particle can be envisioned. However, because the different drugs exhibit sometimes diverging physico-chemical properties (charge and hydrophobicity), a chemical compartmentalization is required for the design of the carrier particle. A rather simple option is to use core-shell nanoparticles, consisting of a core, or inner material surrounded by a shell, or outer layer. ${ }^{18}$ Core-shell nanoparticles 
are widely used for bioimaging ${ }^{19}$ and biosensing, ${ }^{20}$ as well as for other biomedical applications including the co-delivery of drugs. $^{21,22}$ In particular, silica-based core-shell particles are commonly formed from a metal, ${ }^{23,24}$ metal oxide ${ }^{25,26}$ or micellar core covered with a silica shell. ${ }^{27}$ This outer layer gives the particle the same properties as silica particles, such as lower reactivity, enhanced stability in suspension and slower drug release. ${ }^{28}$ Core-shell particles entirely formed by silica have also been reported exhibiting different characteristics such as bimodal pore structures $^{29}$ or selective functionalization of the inner and outer regions of the particles using hybrid sols. ${ }^{30}$

Inspired by the latter approach, we report here a new type of silica core-shell particle having the capability to simultaneously deliver two common topical antibiotics, gentamicin and rifamycin. For this purpose, we have developed a strategy similar to layer-bylayer deposition routes where the positively charged coating of the drugs on the core surface can act as the reactive interface for silica shell deposition. The role of electrostatic and hydrophobic interactions between silanes and antibiotics in the successful synthesis of these core-shell particles is discussed, enlightening the foreseeable versatility of this approach.

\section{Materials and methods}

\subsection{Materials}

Tetraethyl orthosilicate (TEOS, 98\%), (3-aminopropyl)triethoxysilane (APTES, $\geq 98 \%$ ) and (3-mercaptopropyl)trimethoxysilane (MPTMOS, $\geq 95 \%$ ) were purchased from Sigma-Aldrich, as well as the antibiotics sodium rifamycin and gentamicin sulfate. Ammonium hydroxide (30\%) was obtained from Carlo Erba Reagents. All other reagents were of analytical grade and commercially available.

\subsection{Synthesis of silica particles}

2.2.1. Silica particles (SiOH). Bare silica particles were synthesized according to the Stöber method. ${ }^{31}$ Briefly, $21 \mathrm{~mL}$ of tetraethyl orthosilicate were added dropwise to a stirred solution of $32 \mathrm{~mL}$ of ultrapure water, $600 \mathrm{~mL}$ of absolute ethanol, and $45 \mathrm{~mL}$ of ammonium hydroxide. The solution was stirred overnight at room temperature. Particles were washed twice with absolute ethanol, once with deionized water and recovered by centrifugation, dried in a vacuum and stored in a closed flask.

2.2.2. Amino modified silica particles $\left(\mathrm{SiNH}_{2}\right) .1 \mathrm{mmol}$ APTES per gram of $\mathrm{SiOH}$ was added to the reaction medium before washing. The solution was stirred overnight at room temperature and then washed and recovered as described before.

2.2.3. Thiol modified silica particles (SiSH). $5.51 \mathrm{~g}$ of unmodified $\mathrm{SiOH}$ particles were resuspended in a mixture of $548 \mathrm{~mL}$ of absolute ethanol and $12 \mathrm{~mL}$ of ammonium hydroxide, and then $5.3 \mathrm{~mL}$ of MPTMOS was added. It was stirred for 40 minutes at room temperature and then the solvent was evaporated at $80{ }^{\circ} \mathrm{C}$ until $1 / 3$ of the original volume was reached. Particles were washed twice with absolute ethanol, once with deionized water and recovered by centrifugation, dried in a vacuum and stored in a closed flask.
2.2.4. Sulfonate modified silica particles $\left(\mathrm{SiSO}_{3}\right) \cdot 3.6 \mathrm{~g}$ of thiol modified NPs (SiSH) were resuspended in $180 \mathrm{~mL}$ of hydrogen peroxide $35 \%$ and left for 48 hours under stirring. Then the particles were washed three times with ethanol and dried in a vacuum. The power was resuspended and stirred in $150 \mathrm{~mL}$ of sulfuric acid for two hours. Slowly the solution was diluted in deionized water in a cold bath. The particles were recovered and washed with ethanol and deionized water by centrifugation, vacuum dried and stored. ${ }^{32}$

\subsection{Particle loading}

Antibiotic-loaded particles were prepared in deionized water by mixing the particles and the corresponding antibiotic, in a ratio of $375 \mathrm{mg}$ of particles : $20 \mathrm{mg}$ of antibiotic : $50 \mathrm{~mL}$ of deionized water. Suspensions were stirred overnight at room temperature and protected from light. The resulting particles were recovered by centrifugation and washed three times with deionized water. Sodium rifamycin and gentamicin sulfate were used as antibiotics.

\subsection{Preparation of core-shell particles}

The core was synthesized by the Stöber method, modified with thiol or sulfonate for the loading of rifamycin or gentamicin, respectively as described before. The silica shell layer synthesis was carried out with the drug in the ethanol-water medium, in a ratio $500 \mathrm{mg}$ of particles: $27 \mathrm{mg}$ of antibiotics: $0.4 \mathrm{~mL}$ of TEOS : $0.5 \mathrm{~mL}$ of ammonium hydroxide $: 1.5 \mathrm{~mL}$ of deionized water : $50 \mathrm{~mL}$ of ethanol. This shell was modified as described before to achieve core sulfonate-modified and shell thiol-modified particles $\left(\mathrm{SiSO}_{3} @ \mathrm{SiSH}\right)$ or core thiol-modified and shell sulfonatemodified particles ( $\mathrm{SiSH} @ \mathrm{SiSO}_{3}$ ). The second layer was loaded with the second antibiotic (i.e. that was not used in the first step) in the same drug:particle:deionized water ratio. The loaded core-shell particles were then washed and resuspended as described before.

\subsection{Transmission electron microscopy (TEM)}

The size and the shape of the particles were investigated by TEM. Briefly a drop of the sample in aqueous solution was deposited on 400-mesh carbon-coated copper grids. After one minute, the liquid was blotted with filter paper (Whatman no. 4). TEM was performed at room temperature using a JEOL 1011 electron microscope operating at $100 \mathrm{kV}$.

\subsection{Zeta potential}

The zeta potential of the particles was measured using Zeta Plus Brookhaven equipment. For this purpose a $5 \mathrm{mg} \mathrm{mL}^{-1}$ suspension of particles was prepared in $10 \mathrm{mM} \mathrm{KCl}$ aqueous solution. In another experiment, $0.5 \mathrm{mg} \mathrm{mL} \mathrm{m}^{-1}$ of particles were confronted with increasing concentrations of antibiotics. After each step of antibiotic addition, particles were recovered by centrifugation before zeta-potential measurements.

\subsection{Microbiological assay (diffusion method)}

The microbiological assay was carried out using the disk diffusion method as described in the United States Pharmacopeia. Petri dishes with 2 to $5 \mathrm{~mm}$ thick Luria Bertani (LB) agar medium were 
inoculated and homogeneously scattered with $100 \mu \mathrm{L}$ of $1 \times 10^{7} \mathrm{cfu} \mathrm{mL}^{-1}$ in PBS of the bacterial suspension sensitive to the antibiotic of interest. Sterile absorbent paper disks were impregnated with $10 \mu \mathrm{L}$ of the particle suspension and placed on the surface of the agar. Staphylococcus aureus ATCC 29213 (sensitive to rifamycin and gentamicin) and Pseudomonas aeruginosa ATCC 27853 (sensitive to gentamicin) were used. The inhibition zone was measured after incubation at $37{ }^{\circ} \mathrm{C}$ for 24 hours, and evaluated using the corresponding calibration curve of antibiotics selected according to the known sensitivity of the microorganisms to the antibiotic of interest (i.e.: 20-60 $\mu \mathrm{g} \mathrm{mL} \mathrm{m}^{-1}$ for gentamicin and 0.3-1.2 $\mu \mathrm{g} \mathrm{mL}^{-1}$ for rifamycin). In all cases the results are expressed as mean \pm SD from triplicate experiments.

\subsection{Quantification of gentamicin and rifamycin}

The kinetics of the dual drug release behaviour of core-shell particles was determined by Mass Spectrometry. Nanoparticles were suspended at a $30 \mathrm{mg} \mathrm{mL}{ }^{-1}$ concentration in deionized water at $37{ }^{\circ} \mathrm{C}$. At regular intervals of time, they were recovered by centrifugation and the amount of drug released was measured by MS. For this purpose, quantitative and qualitative mass analysis was carried out on a TSQ Quantum Access ${ }^{\mathrm{TM}}$ Max (Thermo Fisher Scientific, San Jose, CA, USA) triple stage quadrupole mass spectrometer with an electrospray ionization (ESI) source and a Rheodyne ${ }^{\mathrm{TM}} 7750 \mathrm{E}-185$ divert/inject valve. Sample injection was performed via the Rheodyne injection valve with a $10 \mu \mathrm{l}$ loop. The linearity of the detector response to sample concentration was assessed by measuring five calibra-

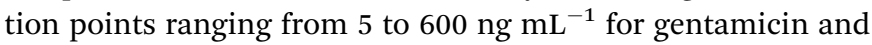
rifamycin. Each calibration point was injected five times. Regression coefficients were obtained by plotting the average peak area versus concentration, using the least squares method (ESI- $1 \dagger$ ). Three ions were selected for the quantification of gentamicin $(\mathrm{m} / \mathrm{z} 464,322$ and 160) and rifamycin (720, 660 and 574).

\subsection{Statistical analysis}

In all cases data are mean $\pm \mathrm{SD}$ of triplicate experiments. The differences were analyzed using two-way ANOVA, followed by the Tukey post-test; $p<0.05$ was considered significant.

\section{Results and discussion}

\subsection{Characterization of surface-modified silica particles}

Monodisperse spherical silica particles were obtained by the Stöber method. ${ }^{31,32}$ The mean diameters for each type of particle were $277 \pm 12 \mathrm{~nm}(\mathrm{SiOH}), 273 \pm 5 \mathrm{~nm}\left(\mathrm{SiNH}_{2}\right), 269 \pm 7 \mathrm{~nm}$ (SiSH) and $277 \pm 2 \mathrm{~nm}\left(\mathrm{SiSO}_{3}\right)$ as determined by TEM (Fig. 1A and $\mathrm{B}$ ), indicating that the particle mean diameter was not significantly affected by the surface modification (ESI- + ). The surface modification was also analyzed by measuring the zeta potential $(\zeta)$ of the particles at different $\mathrm{pH}$ values (Fig. 1C). Starting from $\mathrm{pH} 2$, the bare silica particles $(\mathrm{SiOH})$ are slightly positively charged and undergo a rapid surface charge reversal between pH 3 and 4 before reaching a constant value of $c a .-65 \mathrm{mV}$. For thiol-bearing particles ( $\mathrm{SiSH}$ ), the $\zeta$ value is almost zero in acidic media and reaches $c a$. $-30 \mathrm{mV}$ at $\mathrm{pH}$ 8. After sulfonation, the $\zeta$ value is negative over the whole range of $\mathrm{pH}$, with a slight decay between $\mathrm{pH} 3$ and 5 . Finally, the $\zeta$ value of amino-modified particles $\left(\mathrm{SiNH}_{2}\right)$ follows a similar trend except that values go from a highly positive value $(+65 \mathrm{mV})$ to zero.

The measured evolution for bare silica nanoparticles is in good agreement with the literature, indicating that silanols can exist in three different forms on the silica surface: $\mathrm{SiOH}_{2}{ }^{+}, \mathrm{SiOH}$ and $\mathrm{SiO}^{-}$. The positively charged species are present in a significant amount up to $\mathrm{pH} c a$. 3. This is in agreement with the point of zero charge of 3 for $c a .270 \mathrm{~nm}$ silica particles as reported elsewhere. ${ }^{33}$ Then it was suggested that further deprotonation involves two populations of silanol groups, $20 \%$ being acidic $\left(\mathrm{p} K_{\mathrm{a}} \mathrm{SiOH} / \mathrm{SiO}^{-}=4.5\right)$ and $80 \%$ basic $\left(\mathrm{p} K_{\mathrm{a}} \mathrm{SiOH} / \mathrm{SiO}^{-}=8.5\right)$. In the case of thiol groups, with a $\mathrm{p} K_{\mathrm{a}}\left(\mathrm{SH} / \mathrm{S}^{-}\right)$of $c a .10 .5$, the organic function should not contribute to the surface charge of the
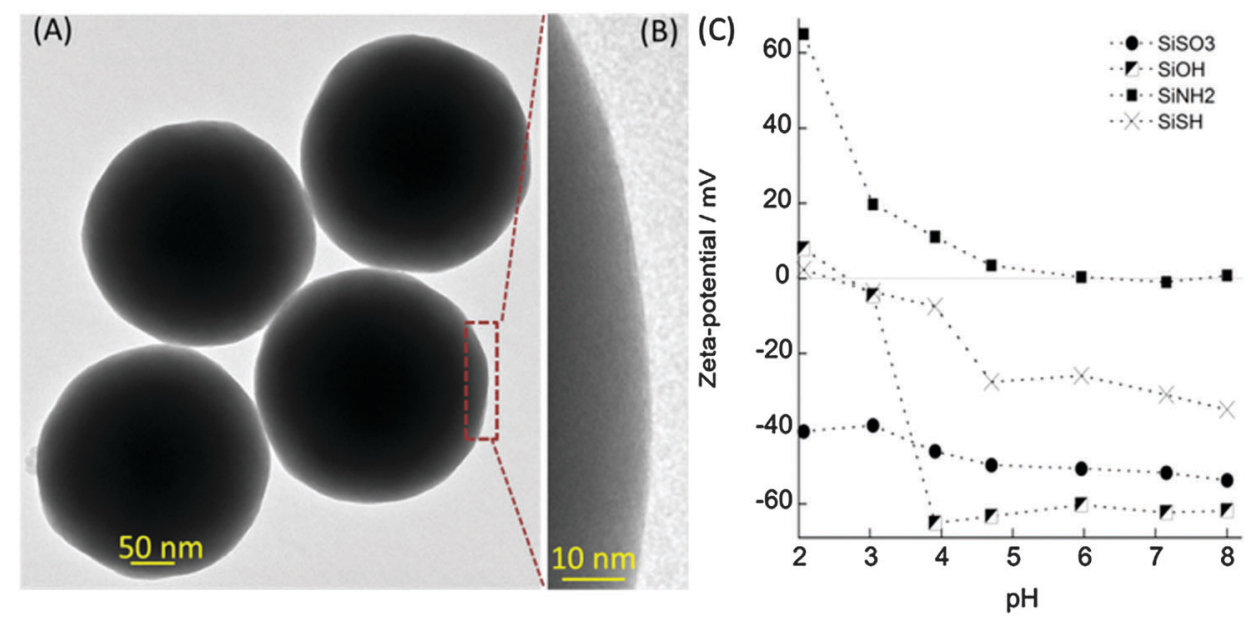

Fig. 1 (A and B) TEM photo of unmodified SiOH particles. (C) Zeta-potential of sulfonate-modified ( $\left.\mathrm{SiSO}_{3}\right)$, unmodified ( $\left.\mathrm{SiOH}\right)$, amino-modified (SiNH 2 ) and thiol-modified (SiSH) silica particles at different $\mathrm{pH}$ values $(10 \mathrm{mM} \mathrm{KCl})$. 
particle in the investigated $\mathrm{pH}$ range. Therefore the observed $\zeta$ evolution can be attributed to the decrease of available silanol groups on the particle surface after grafting, leading to a lower density of $\mathrm{SiOH}_{2}{ }^{+}$in acidic media and $\mathrm{SiO}^{-}$in basic solution, therefore narrowing the range of $\zeta$ variation. After sulfonation, the strongly acidic $\mathrm{SO}_{3}{ }^{-}$groups $\left(\mathrm{pK}_{\mathrm{a}} \mathrm{SO}_{3} \mathrm{H} / \mathrm{SO}_{3}{ }^{-}<1\right)$ should contribute to a constant negative charge that can be evidenced at low $\mathrm{pH}$. However, in basic medium, the absolute value of $\zeta$ for sulfonated particles is smaller than that for bare silica particles. This indicates that, from a surface charge point of view, the decrease in the number of free silanol groups on the silica surface that results from the grafting reaction is not compensated by the presence of sulfonate groups on the organic chain of the silane. Accordingly, since $\mathrm{p} K_{\mathrm{a}}$ of primary amines is $c a$. 10.5, their protonation degree should be constant over the 2-8 $\mathrm{pH}$ range of this study and should therefore contribute in a constant manner to the surface charge of $\mathrm{SiNH}_{2}$ particles. Comparison of the $\zeta$ evolution of $\mathrm{SiNH}_{2}$ and $\mathrm{SiOH}$ shows that this is indeed the case except in the 3-4 $\mathrm{pH}$ range. This probably reflects the existence of direct acid-base reactions between surface silanolate and ammonium groups from APTES, as reported in the literature. ${ }^{34}$

\subsection{Characterization of antibiotic-coated silica particles}

Silica particles were loaded with two oppositely charged antibiotics, gentamicin being positively charged, while rifamycin is negatively charged. TEM analysis revealed that the particle mean diameter was not significantly affected by the antibiotic adsorption (ESI-3†). Based on preliminary experiments, particle loading was performed in a large excess of antibiotics $20 \mathrm{mg}$ of antibiotics for $375 \mathrm{mg}$ of particles in water). After washing, the gentamicin and rifamycin loadings were evaluated on $P$. aeruginosa and $S$. aureus, respectively. Antibiograms were obtained using the disk diffusion method, and inhibition zones were measured after incubation for $24 \mathrm{~h}$ at $37{ }^{\circ} \mathrm{C}$. After this period, impregnated paper discs were transferred to new agar media seeded with bacteria and incubated for an additional $24 \mathrm{~h}$ period. However, no antibacterial activity could be observed after this delay, suggesting that a major fraction of the antibiotics was released over the first $24 \mathrm{~h}$. Unloaded particles showed no antibacterial activity in all cases indicating that the measured bactericidal effects originated from the drugs only.

In the case of gentamicin, the largest antibiotic amount was found for the most negatively charged particles $\mathrm{SiOH}$ and $\mathrm{SiSO}_{3}{ }^{-}$compared to thiol-( $\left.\mathrm{SiSH}\right)$ and amino- $\left(\mathrm{SiNH}_{2}\right)$ modified silica particles (Fig. 2). This suggests that attractive electrostatic interactions are the main driving force for the sorption process. Indeed, the amounts of gentamicin in $\mathrm{SiOH}$ and $\mathrm{SiSO}_{3}{ }^{-}$ are $1509 \mu \mathrm{g} \mathrm{g}^{-1}$ and $1793 \mu \mathrm{g} \mathrm{g}^{-1}$, respectively, which are 6 and 7 times higher than the amount found in SiSH (Table 1).

On the other hand, in the case of the negatively charged rifamycin, the situation is more complex. Despite their positively charged surface, $\mathrm{SiNH}_{2}$ particles do not adsorb significantly more antibiotic than bare particles. Moreover, the highest rifamycin loading was obtained for slightly negatively charged SiSH particles with $19.2 \mu \mathrm{g} \mathrm{g}^{-1}$ (Table 1). This can be explained by considering that rifamycin is classified as an hydrophobic molecule, with an octanol-water partition coefficient of $2.77,{ }^{35}$ while thiol groups have the capability to confer non-polar-properties to modified surfaces. ${ }^{36,37}$ Therefore it is very likely that hydrophobic interactions are involved in the rifamycin sorption on SiSH. Sulfonated particles were not evaluated as their negative surface charge was expected to repel anionic rifamycin molecules.

To clarify the sorption process, the evolution of zeta potential with antibiotic concentration was studied for the antibioticloaded particles that exhibited even a low antimicrobial activity, namely $\mathrm{SiSO}_{3}, \mathrm{SiOH}$, and $\mathrm{SiSH}$ for gentamicin, and $\mathrm{SiNH}_{2}$, and $\mathrm{SiSH}$ for rifamycin. In the case of gentamicin adsorption, a gradual increase in the $\zeta$ value with antibiotic concentration was observed for $\mathrm{SiOH}, \mathrm{SiSO}_{3}{ }^{-}$and $\mathrm{SiSH}$ up to slightly positive values $(+5 /+15 \mathrm{mV}$, Fig. 3$)$. The difference in the $\zeta$ value between uncoated and saturated surfaces for each type of particle nicely correlates with the drug loading as determined by the disk method. Altogether, this supports the previous hypothesis that the sorption process is driven by attractive electrostatic interactions and that saturation occurs after neutralization of the negative charge of the particle surface by positively charged gentamicin. Nevertheless, it must be noticed that the final $\zeta$ value is slightly positive. This suggests that a fraction of the antibiotic is adsorbed via other interactions either with the silica
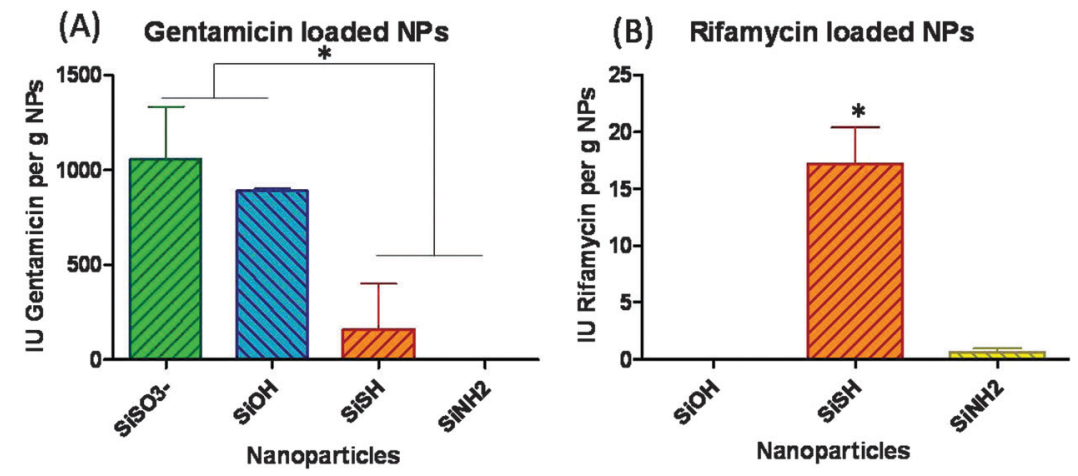

Fig. 2 Disk diffusion method evaluation of the antibacterial activity. Activities expressed as International Units (IUs) of the drug per gram of particles: (A) gentamicin and (B) rifamycin. The results are expressed as mean \pm SD from at least triplicate experiments. * indicates the statistical significant difference $(p<0.0001)$. 
Table 1 IU drug per gram and the corresponding $\mu \mathrm{g}$ per gram of (A) gentamicin and (B) rifamycin loaded particles. The results are expressed as mean \pm SD from at least triplicate experiments

\begin{tabular}{lcc}
\hline & IU Drug per $g$ of NPs & $\mu$ g of drug per $g$ of NPs \\
\hline (A) Gentamicin & & \\
SiSO $_{3}{ }^{-}$ & $1058 \pm 256$ & $1793 \pm 467$ \\
SiOH & $890 \pm 11$ & $1509 \pm 20$ \\
SiSH & $160 \pm 240$ & $271 \pm 407$ \\
SiNH $_{2}$ & $0 \pm 0$ & $0 \pm 0$ \\
(B) Rifamycin & & \\
SiSH & $17.2 \pm 3.2$ & $19.2 \pm 3.5$ \\
SiNH $_{2}$ & $0.6 \pm 0.3$ & $0.7 \pm 0.4$ \\
SiOH & $0.0 \pm 0.0$ & $0.0 \pm 0.0$ \\
\hline
\end{tabular}

surface or with already-deposited molecules. In particular, the presence of three hydroxyl groups on the gentamicin backbone should favor hydrogen bond formation.

In the case of rifamycin, the relationship between zeta-potential and drug adsorption is not straightforward. The addition of a small amount of antibiotic leads to a decrease of $\zeta$ for both $\mathrm{SiSH}$ and $\mathrm{SiNH}_{2}$. This event is more pronounced for the former than for the latter, suggesting that it corresponds to the adsorption of the negatively charged antibiotics. Increasing further the antibiotic concentration does not significantly modify the $\zeta$ value, but a slight continuous increase is obtained for $\mathrm{SiSH}$. Since the rifamycin loading capacity of these two systems is about hundred times lower than for gentamicin (see Table 1), it can be expected that surface saturation is reached at such low concentrations. Moreover, near neutral $\mathrm{pH}$, gentamicin bears five positive charges per molecule, whereas rifamycin has only one negatively charged group so that the former should have more influence on the overall particle surface charge than the latter at a similar surface concentration (Fig. 4). As a matter of fact, the two drugs also differ from the point of view of the accessibility of the ionized groups. In the gentamicin structure, the ammonium functions point out of the glycosidic rings, whereas the $\mathrm{OH}$ group of rifamycin belongs to a naphthalene ring inducing a sterical barrier and conferring a strong hydrophobic character to the molecule. This can explain its low sorption on $\mathrm{SiNH}_{2}$ particles despite their high positive charge. It also strengthens our hypothesis about the key role of hydrophobic interactions in rifamycin sorption.

\subsection{Core-shell nanoparticles and their dual antibiotic drug release properties}

In a step forward, particles capable of releasing two antibiotics were designed. Our strategy involves the synthesis and functionalization of a Stöber silica core and its loading with the first antibiotic (drug A), followed by the deposition of the shell that is further functionalized and loaded with the second antibiotic (drug B) (Fig. 5). The evolution of the particle size, as determined by DLS, and of their surface charge, as obtained by $\zeta$
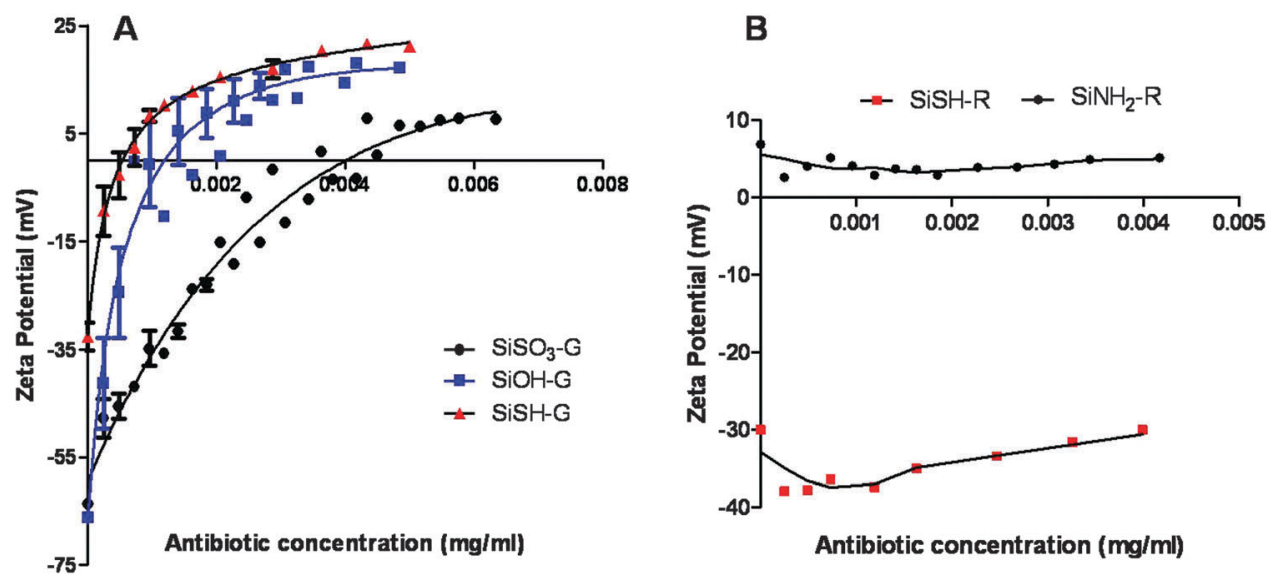

Fig. 3 Zeta-potential of silica particles as a function of antibiotic concentration. (A) Gentamicin-loaded sulfonate (SiSO $\left.{ }_{3}-\mathrm{G}\right)$, unmodified ( $\left.\mathrm{SiOH}-\mathrm{G}\right)$, and thiol (SiSH-G) modified particles. (B) Rifamycin loaded amino $\left(\mathrm{SiNH}_{2}-\mathrm{R}\right)$ and thiol (SiSH-R) modified particles.<smiles>CN[C@@H](C)C1CCC(N)[C@H](OC2[C@H](N)C[C@H](N)[C@@H](OC3OC[C@](C)(O)[C@H](NC)[C@H]3O)[C@@H]2O)O1</smiles>

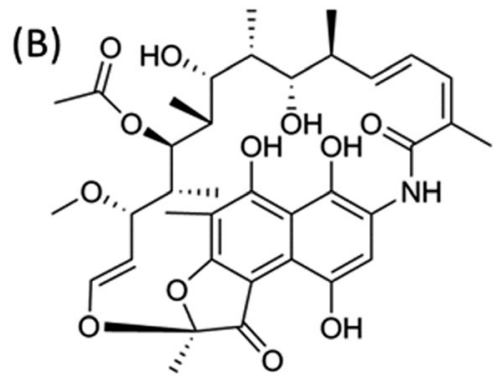

Fig. 4 Chemical structures of (A) gentamicin and (B) rifamycin. 


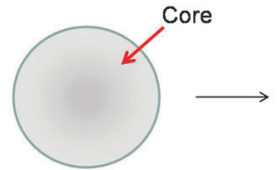

Core synthesis (First Stöber)

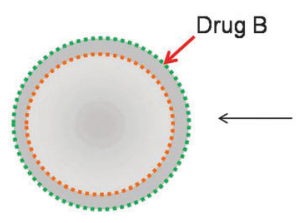

Drug B loading
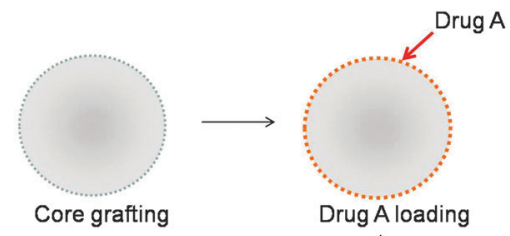

Drug A loading

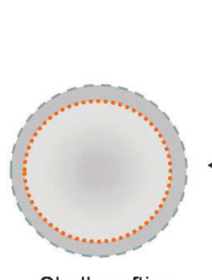

Shell grafting

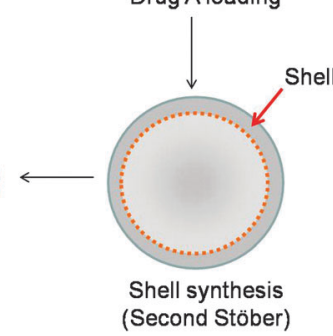

(Second Stöber)
Fig. 5 Scheme of the synthesis of double drug-loaded core-shell particles based on a two step Stöber process.

measurements, are provided in Table 2 at each step of the preparation process.

A first configuration was studied using the sulfonated core loaded with gentamicin, as it exhibited the higher drug loading, further coated with a silica shell and functionalized with thiol groups followed by rifamycin sorption ( $\mathrm{SiSO}_{3}-\mathrm{G} @ \mathrm{SiSH}-\mathrm{R}$ ). $\zeta$ data show that the adsorption of gentamicin on $\mathrm{SiSO}_{3}$ leads to a decrease in negative charge of the particles, as expected for the deposition of the cationic antibiotic. DLS also indicates a slight increase in the particle diameter. Note that these data were obtained after rinsing the particles so that, compared to the values obtained in the presence of an excess of antibiotics and representative of the synthesis process (Fig. 3), partial gentamicin desorption has occurred leading to a negative value of $\zeta$. Subsequently, reaction of $\mathrm{SiSO}_{3}-\mathrm{G}$ with TEOS leads to an increase of the absolute value of $\zeta$, reaching $c a$. $-60 \mathrm{mV}$ and therefore close to the value of bare $\mathrm{Si}-\mathrm{OH}$ particles, strongly supporting the formation of a silica shell. In parallel, DLS data also suggest an increase in particle diameter but the relatively high value of the standard deviation for $\mathrm{SiSO}_{3}-\mathrm{G}(40 \mathrm{~nm})$ does not allow the calculation of the shell thickness. After outer grafting with MPTMOS, the $\zeta$ decreases in absolute value, similar to our previous observation of MPTMOS reaction on core silica particles. The successful formation and functionalization of the shell were also supported by analyzing the $\mathrm{pH}$ dependence of the $\zeta$ value of the particles after synthesis (Fig. 6). The evolution of the zeta potential in the $\mathrm{pH}$ range of 2 to 8 closely follows that of the thiolated core particles, indicating that the resulting surface chemistry is similar. Finally, further contact with rifamycin decreases again the absolute value of $\zeta$.

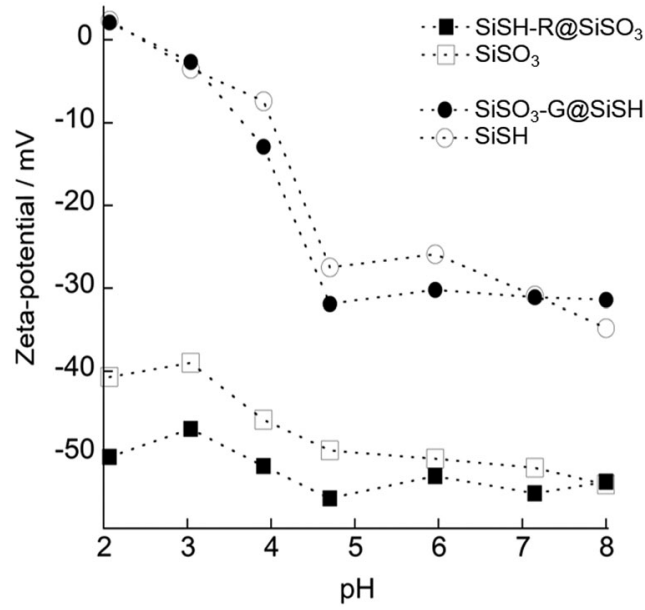

Fig. 6 Zeta-potential of silica particles and core-shell particles as a function of pH: $\mathrm{SiSH}-\mathrm{RaSiSO}{ }_{3}$ as compared with $\mathrm{SiSO}_{3}$ and $\mathrm{SiSO}_{3}-\mathrm{GaSH}$ as compared with $\mathrm{SiSH}$.

In parallel, DLS data suggest an important aggregation of the particles.

The mirror situation using the thiolated core loaded with rifamycin coated with a silica shell and functionalized with sulfonated groups, followed by gentamicin sorption, (SiSH-R@) $\mathrm{SiSO}_{3}-\mathrm{G}$ ) was studied. As seen in Table 2, after contact of TEOS with the rifamycin-coated thiolated core particles, only a slight variation of $\zeta$ is measured for the expected (SiSH-R@SiOH) system and this value is not significantly modified after MPTMOS grafting and sulfonation (SiSH-R@SiSO ${ }_{3}$ ). In parallel all particle sizes obtained from DLS were within the standard deviation range, except for the final gentamicin deposition. A more detailed study of the $\mathrm{pH}$-dependence of the $\zeta$ for $\mathrm{SiSH}-\mathrm{R} @ \mathrm{SiSO}_{3}$ systems shows that it follows that of sulfonated cores but with a significantly more negative value under acidic conditions (Fig. 6). This suggests that the oxidation reaction required for sulfonation of thiol groups of the shell also impacts the thiol groups present on the core particle. In addition, the experimental conditions for the sulfonation of the shell are particularly harsh when considering the shell formation of preloaded cores, involving a multistep process with hydrogen peroxide, sulfuric acid and successive washings. This suggests that such a combination is not suitable to obtain bi-functional core-shell particles so that only gentamicin loading of the core will be considered for the rest of the manuscript.

The structure of the particles obtained with and without antibiotics was then analyzed by TEM. As can be seen in Fig. 7, for antibiotic-free core particles, no significant modification of

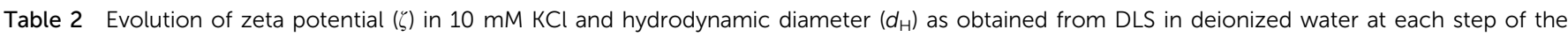
bifunctional core-shell particle synthesis (G: gentamicin; R: rifamycin). The results are expressed as mean \pm SD from at least triplicate experiments

\begin{tabular}{lllll}
\hline Sample & $\zeta(\mathrm{mV})$ & $d_{\mathrm{H}}(\mathrm{nm})$ & Sample & $\zeta(\mathrm{mV})$ \\
\hline $\mathrm{SiSO}_{3}$ & $-50 \pm 3$ & $257 \pm 16$ & SiSH & $-38 \pm 2$ \\
$\mathrm{SiSO}_{3}$-G & $-18 \pm 1$ & $325 \pm 42$ & SiSH-R & $-32 \pm 2$ \\
$\mathrm{SiSO}_{3}$-G@SiOH & $-59 \pm 2$ & $358 \pm 17$ & SiSH-R@SiOH & $-55 \pm 2$ \\
SiSO $_{3}$-G@SiSH & $-32 \pm 1$ & $377 \pm 13$ & SiSH-R@SiSO & $258 \pm 7$ \\
SiSO $_{3}$-G@SiSH-R & $-13 \pm 2$ & $1240 \pm 157$ & SiSH-R@SiSO & -4 \\
\end{tabular}




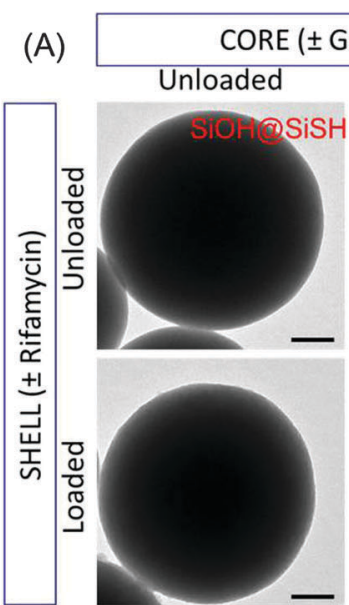

(B)
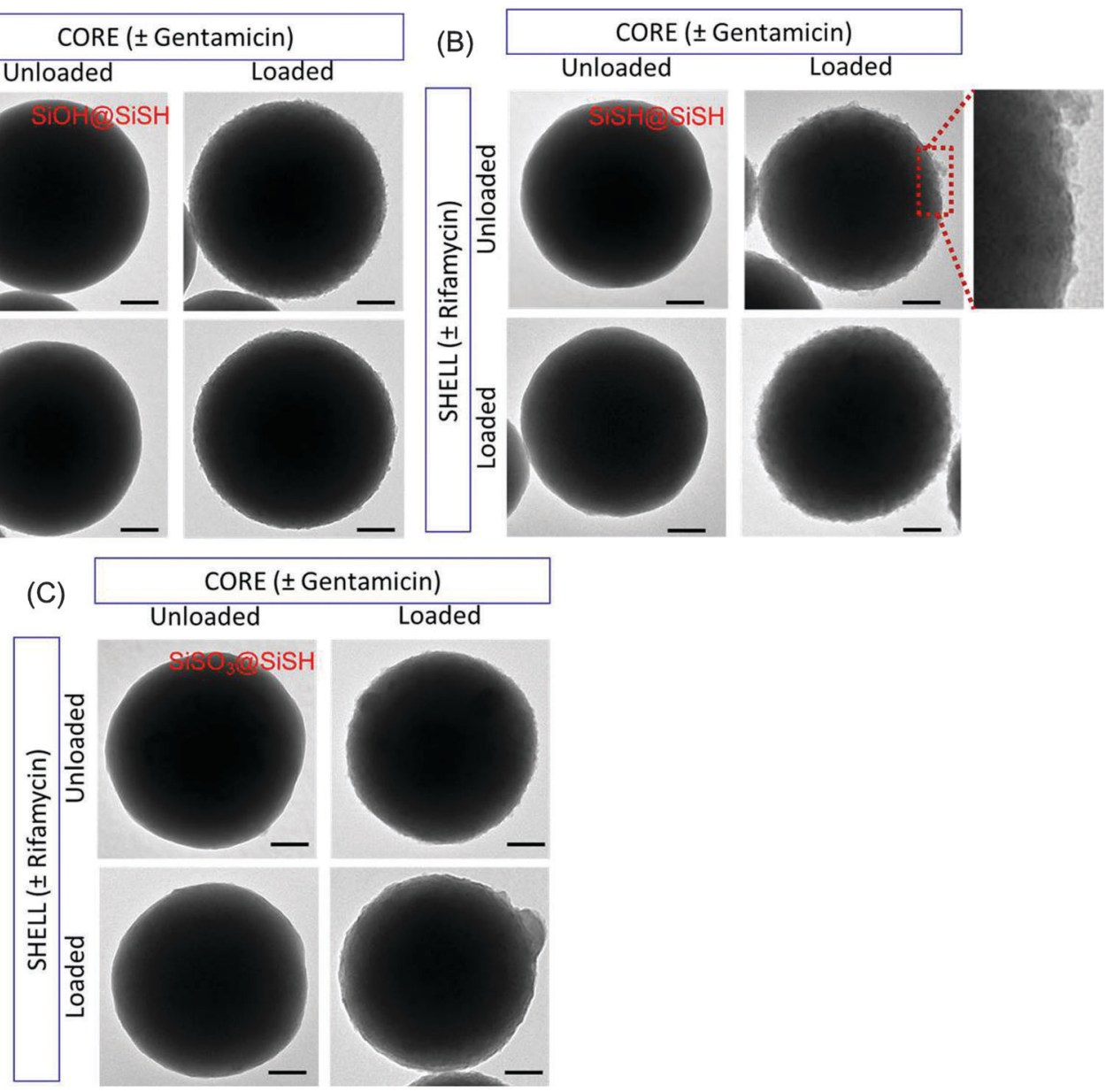

Fig. 7 TEM images of: (A) SiOH@SiSH, (B) SiSH@SiSH and (C) $\mathrm{SiSO}_{3} @$ SiSH core-shell particles, with or without gentamicin (G)-loaded cores and with or without rifamycin (R)-loaded shells (scale bar $50 \mathrm{~nm}$ ).

the surface of the final colloids was observed, even after the sorption of the second drug. In contrast, when gentamicin was initially deposited on the core particle, an additional thin layer was observed on the particle surface. This layer could still be observed after further shell surface sorption of rifamycin.

The deposition of a silica shell was confirmed by SEM imaging (Fig. 8 and ESI- $4 \dagger$ ). The surface of the silica shell grown on the surface of gentamicin-coated $\mathrm{SiSO}_{3}$ particles

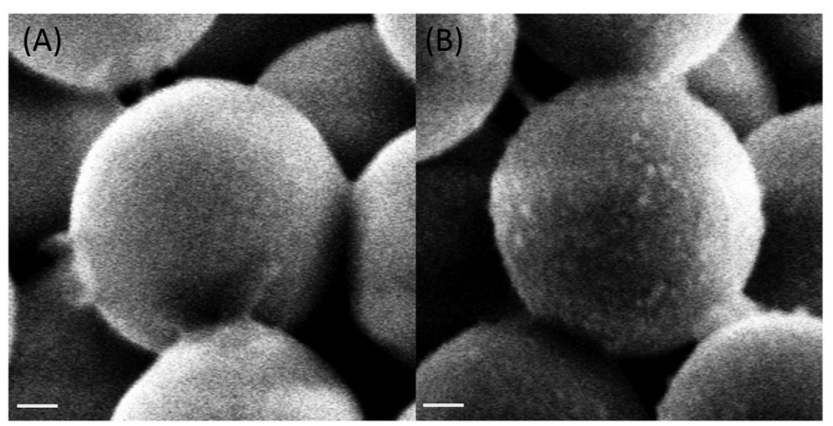

Fig. $8 \mathrm{SEM}$ images of (A) $\mathrm{SiSO}_{3} \mathrm{CSiSH}$ and (B) $\mathrm{SiSO}_{3}-\mathrm{GaSiSH}-\mathrm{R}$ particles (scale bar $50 \mathrm{~nm}$ ). shows a granular aspect that is also strikingly observed after rifamycin sorption on these core-shell systems.

At this point, it is important to consider the shell process formation. The possibility to form $\mathrm{SiO}_{2}$ shells on silica particles under the Stöber conditions has been widely described. ${ }^{38}$ Although electrostatic interactions are unfavorable for silica deposition on bare silica particles, it may proceed thanks to the promotion of the condensation reaction of silanols and silonates under basic conditions. Surface modification by cationic coating introduces favorable electrostatic interactions promoting silica deposition. ${ }^{39}$ In contrast, the introduction of anionic groups on the particle surface should limit shell formation. The effect of grafting hydrophobic moieties should also make the coating process of hydrophilic silica less favorable than for bare particles. Coming back to our samples, the efficiency of the shell layer deposition of silica particles should vary as $\mathrm{SiOH}>\mathrm{SiSH}>\mathrm{SiSO}_{3}$. Noticeably, TEM images could not provide any evidence that such a reaction occurred under these conditions. However, this situation was changed after gentamicin sorption as the surface turned positive, favoring the formation of an observable silica shell. On this basis, only the $\mathrm{SiSO}_{3} @ \mathrm{SiSH}$ configuration was further studied. 
Antimicrobial activity against the two bacterial strains (gentamicin-sensitive $P$. aeruginosa and gentamicin-rifamycinsensitive $S$. aureus) was achieved when the two drugs were present within the core-shell particles. The diameters of the corresponding inhibition zones were taken as $100 \%$ to compare the efficiency of these dual systems with particles containing a single antibiotic (Fig. 9). For both bacteria, drug-free coreshell particles showed no significant antibacterial activity. For $P$. aeruginosa, the gentamicin-free particles were ineffective whereas the gentamicin-loaded particles showed an antibacterial activity similar to the particles with both antibiotics. This is in good agreement with the fact that rifamycin is poorly effective towards Gram-negative bacteria. It is important to point out that gentamicin-coated core particles and core-shell systems have a similar antibacterial efficiency, indicating that no important leaching of gentamicin occurred from the particle core during rifamycin sorption on the shell. For $S$. aureus, the core-shell particles containing both drugs presented an antimicrobial activity 1.5 times higher than the two systems containing only one antibiotic. These results indicate that these core-shell particles can efficiently deliver both antibiotics.

To clarify this point, mass spectrometry analysis of a particle suspension supernatant allowed us to identify the presence of gentamicin and rifamycin ${ }^{40,41}$ (ESI-5 $\dagger$ ) and further confirmed the release of both antibiotics from the core-shell particles. The kinetics profiles revealed that $43 \%$ of the rifamycin is released during the first 30 minutes while the release of $50 \%$ of the gentamicin requires a four time longer incubation time. Moreover, rifamycin is completely released from the particles within 3 hours while only $71 \%$ of the gentamicin is released over the same period (Fig. 10). This is in agreement with the proposed mechanism where the outer rifamycin coating is rapidly desorbed whereas gentamicin release requires the progressive diffusion through the silica shell or dissolution of the silica shell.

Our procedure has some similarities with layer-by-layer routes, where the core-shell structure is built up from the alternation of coatings with opposite charges. Whereas a close strategy was previously described to build up bi-functional core-shell mesoporous particles using hybrid sols, ${ }^{30}$ we have here taken advantage of the antibiotic layer itself as a charged interface for shell deposition, limiting its leaching during silica formation and further chemical modification. This strategy should therefore be applicable to any positively charged drug as a core component, whereas the shell coating may be of various natures. Importantly, although the mirror situation, i.e. direct shell coating from TEOS on a negatively charged core, does not appear to be possible as such, the use of hybrid sols containing cationic silanes, such as TEOS/APTES mixtures, should allow for further silica deposition. ${ }^{42}$ Accordingly, the use of hydrophobic sols can favor shell formation on the surface of core particles coated with lipophilic drugs. ${ }^{43}$

This strategy can also be extended to mesoporous particles that may offer the possibility for the loading of up to four drugs (inside core, outside core, inside shell, and outside shell) whose
(A)

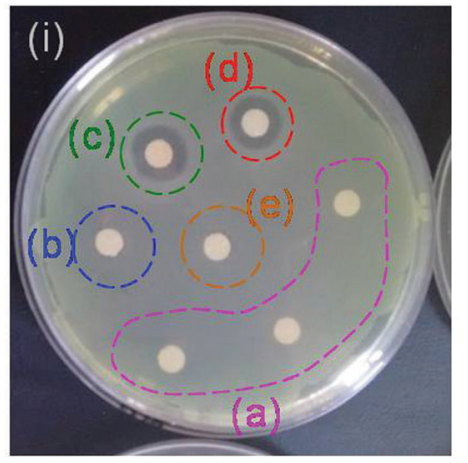

(B) (i)

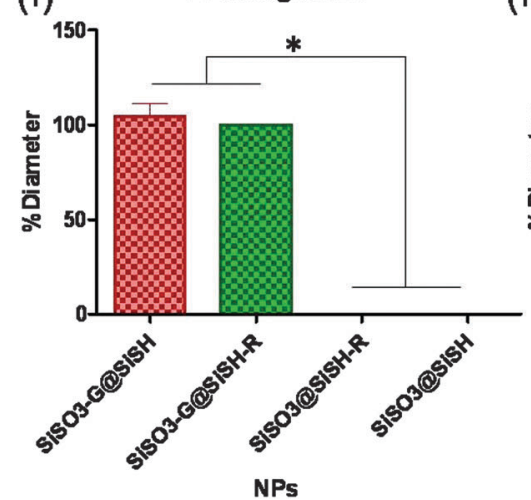

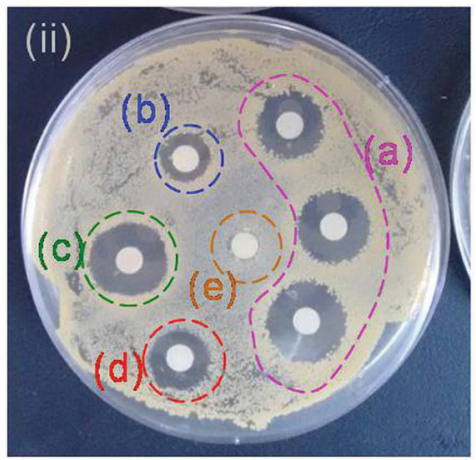

(ii)

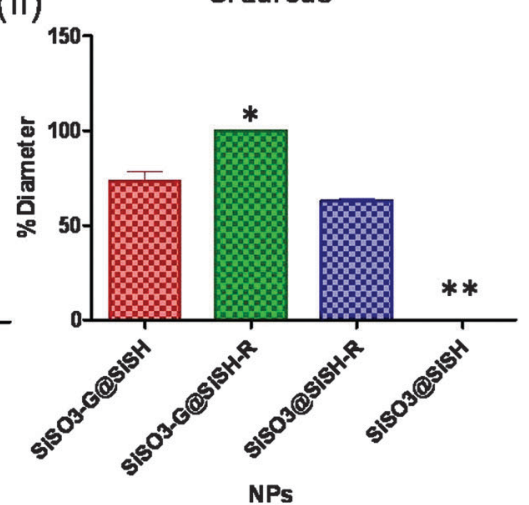

Fig. 9 Disk diffusion method evaluation of the antibacterial activity of core-shell particles: (A) plates obtained with (a) rifamycin calibration curve $\left(0.3-0.6-1.2 \mu \mathrm{g} \mathrm{mL}^{-1}\right.$ ), (b) $\mathrm{SiSO}_{3} \mathrm{QSiSH}-\mathrm{R}$, (c) $\mathrm{SiSO}_{3}-\mathrm{GaSiSH}-\mathrm{R}$, (d) $\mathrm{SiSO}_{3}-\mathrm{G}\left(\mathrm{SSiSH}\right.$ and (e) $\mathrm{SiSO}_{3} \mathrm{QSiSH}$ on (i) P. aeruginosa and (ii) S. aureus. (B) Corresponding diameter size relative to $\mathrm{SiSO}_{3}-\mathrm{G}(\mathrm{aSiSH}-\mathrm{R}(100 \%)$ for (i) $P$. aeruginosa and (ii) S. aureus. * and ** indicate the statistical significant difference ( $p<0.0001)$. 


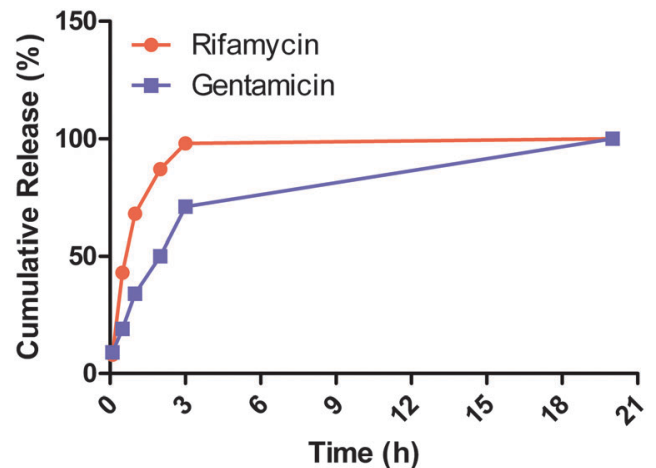

Fig. 10 Dual release profiles of rifamycin and gentamicin from the core-shell particles measured by mass spectrometry.

sequential release may be additionally tuned by gate-keeping approaches. ${ }^{44-46}$ Such a strategy may be of particular interest in so-called combination therapy that has become a key strategy in cancer treatment. ${ }^{47}$

\section{Conclusions}

The co-encapsulation of drugs with different and sometimes divergent, physico-chemical properties within a single carrier requires the development of compartmentalization strategies. The core-shell approach appears to be particularly suitable for this purpose but faces possible interference in the presence of drugs associated with the core particle with further deposition of the shell. In contrast, we show here that it is possible to benefit from the presence of a positively charged antibiotic coating to promote further silica deposition. This approach also has the advantage to avoid premature leaching of the core drug during shell formation. As a step further, it is now important to extend this layer-by-layer strategy to anionic and hydrophobic drugs. In addition, further control of the shell structure should allow for a temporally differentiated control of the release kinetics of the two molecules.

\section{Acknowledgements}

A. M. Mebert is grateful for her doctoral fellowship granted by the National Research Council (CONICET). Y. Shi is funded by the China Scholarship Council. The authors would like to acknowledge the support of grants from the Universidad de Buenos Aires UBACYT 20020110100081, from CONICET PIP 11220120100657CO and from Agencia Nacional de Investigaciones Cientificas y Técnicas PICT 2012-1441 (to M. F. D). M. F. D. and T. C. thank the Argentina-France MINCYT-ECOS-Sud (project A12S01) and CONICET-CNRS programs for financial support of their collaboration. The authors would like to thank J. Nesterzak for his technical assistance.

\section{References}

1 G. Tiwari, R. Tiwari, B. Sriwastawa, L. Bhati, S. Pandey, P. Pandey and S. K. Bannerjee, Int. J. Pharm. Invest., 2012, 2, $2-11$.
2 N. J. Medlicott, M. J. Rathbone, I. G. Tucker and D. W. Holborow, Adv. Drug Delivery Rev., 1994, 13, 181-203.

3 I. Hornyxak, E. Madacsi, P. Kalugyer, G. Vacz, D. B. Horvathy, M. Szendroi, W. Han and Z. Lacza, BioMed Res. Int., 2014, 2014, 8 .

4 S. J. McConoughey, R. P. Howlin, J. Wiseman, P. Stoodley and J. H. Calhoun, J. Biomed. Mater. Res., Part B, 2015, 103, 870-877.

5 A. R. Short, D. Koralla, A. Deshmukh, B. Wissel, B. Stocker, M. Calhoun, D. Dean and J. O. Winter, J. Mater. Chem. B, 2015, 3, 7818-7830.

6 A. Saito, H. Miyazaki, T. Fujie, S. Ohtsubo, M. Kinoshita, D. Saitoh and S. Takeoka, Acta Biomater., 2012, 8, 2932-2940.

7 T. Bollenbach, Curr. Opin. Microbiol., 2015, 27, 1-9.

8 A. Santos, M. Sinn Aw, M. Bariana, T. Kumeria, Y. Wang and D. Losic, J. Mater. Chem. B, 2014, 2, 6157-6182.

9 M. Zilberman, D. Egozi, M. Shemesh, A. Keren, E. Mazor, M. Baranes-Zeevi, N. Goldstein, I. Berdicevsky, A. Gilhar and Y. Ullmann, Acta Biomater., 2015, 22, 155-163.

10 G. S. Alvarez, C. Helary, A. M. Mebert, X. Wang, T. Coradin and M. F. Desimone, J. Mater. Chem. B, 2014, 2, 4660-4670.

11 B. Song, C. Wu and J. Chang, Acta Biomater., 2012, 8, 1901-1907.

12 Y. Wang, A. Santos, A. Evdokiou and D. Losic, J. Mater. Chem. B, 2015, 3, 7153-7172.

13 H. Zhang, D. R. Dunphy, X. Jiang, H. Meng, B. Sun, D. Tarn, M. Xue, X. Wang, S. Lin, Z. Ji, R. Li, F. L. Garcia, J. Yang, M. L. Kirk, T. Xia, J. I. Zink, A. Nel and C. J. Brinker, J. Am. Chem. Soc., 2012, 134, 15790-15804.

14 D. Lison, L. C. J. Thomassen, V. Rabolli, L. Gonzalez, D. Napierska, J. W. Seo, M. Kirsch-Volders, P. Hoet, C. E. A. Kirschhock and J. A. Martens, Toxicol. Sci., 2008, 104, 155-162.

15 D. Napierska, L. C. J. Thomassen, V. Rabolli, D. Lison, L. Gonzalez, M. Kirsch-Volders, J. A. Martens and P. H. Hoet, Small, 2009, 5, 846-853.

16 S. Quignard, G. Mosser, M. Boissière and T. Coradin, Biomaterials, 2012, 33, 4431-4442.

17 C. G. Gonzalez, G. S. Álvarez, D. E. Camporotondi, M. L. Foglia, C. Aimé, L. E. Diaz, T. Coradin and M. F. Desimone, Silicon, 2014, DOI: 10.1007/s12633-12014-19203-12635.

18 R. Ghosh Chaudhuri and S. Paria, Chem. Rev., 2012, 112, 2373-2433.

19 T.-J. Yoon, K. N. Yu, E. Kim, J. S. Kim, B. G. Kim, S.-H. Yun, B.-H. Sohn, M.-H. Cho, J.-K. Lee and S. B. Park, Small, 2006, 2, 209-215.

20 I. Al-Ogaidi, H. Gou, A. K. A. Al-kazaz, Z. P. Aguilar, A. K. Melconian, P. Zheng and N. Wu, Anal. Chim. Acta, 2014, 811, 76-80.

21 Y. Wang, S. Gao, W.-H. Ye, H. S. Yoon and Y.-Y. Yang, Nat. Mater., 2006, 5, 791-796.

22 G.-F. Luo, W.-H. Chen, Y. Liu, Q. Lei, R.-X. Zhuo and X.-Z. Zhang, Sci. Rep., 2014, 4, 6064.

23 H. U. Lee, C. Park and S. W. Kim, Process Biochem., 2012, 47, 1282-1286.

24 S. C. Larsen, S. E. Lehman, A. S. Morris, P. S. Mueller, A. Salem and V. Grassian, Environ. Sci.: Nano, 2016, 3, 56-66. 
25 A. A. Ansari and M. Alam, J. Lumin., 2015, 157, 257-263.

26 F. Jiang, Y. Fu, Y. Zhu, Z. Tang and P. Sheng, J. Alloys Compd., 2012, 543, 43-48.

27 J. Liu, C. Detrembleur, S. Mornet, C. Jérôme and E. Duguet, J. Mater. Chem. B, 2015, 3, 6117-6147.

28 Q. Huo, J. Liu, L.-Q. Wang, Y. Jiang, T. N. Lambert and E. Fang, J. Am. Chem. Soc., 2006, 128, 6447-6453.

29 H. Ishii, T. Ikuno, A. Shimojima and T. Okubo, J. Colloid Interface Sci., 2015, 448, 57-64.

30 V. Cauda, A. Schlossbauer, J. Kecht, A. Zürner and T. Bein, J. Am. Chem. Soc., 2009, 131, 11361-11370.

31 L. C. J. Thomassen, A. Aerts, V. Rabolli, D. Lison, L. Gonzalez, M. Kirsch-Volders, D. Napierska, P. H. Hoet, C. E. A. Kirschhock and J. A. Martens, Langmuir, 2010, 26, 328-335.

32 C. Aimé, G. Mosser, G. Pembouong, L. Bouteiller and T. Coradin, Nanoscale, 2012, 4, 7127-7134.

33 S. V. Patwardhan, F. S. Emami, R. J. Berry, S. E. Jones, R. R. Naik, O. Deschaume, H. Heinz and C. C. Perry, J. Am. Chem. Soc., 2012, 134, 6244-6256.

34 R. G. Acres, A. V. Ellis, J. Alvino, C. E. Lenahan, D. A. Khodakov, G. F. Metha and G. G. Andersson, J. Phys. Chem. C, 2012, 116, 6289-6297.
35 W. H. Cover and S. C. Rittenberg, J. Bacteriol., 1984, 157, 391-397.

36 H. Niu, S. Wang, T. Zeng, Y. Wang, X. Zhang, Z. Meng and Y. Cai, J. Mater. Chem., 2012, 22, 15644-15653.

37 M. H. Marchena, M. Granada, A. V. Bordoni, M. Joselevich, H. Troiani, F. J. Williams and A. Wolosiuk, J. Solid State Chem., 2012, 187, 97-102.

38 A. van Blaaderen and A. Vrij, Langmuir, 1992, 8, 2921-2931.

39 T. Coradin, E. Mercey, L. Lisnard and J. Livage, Chem. Commun., 2001, 2496-2497.

40 N. Isoherranen and S. Soback, Analyst, 2000, 125, 1573-1576.

41 B. Prasad and S. Singh, J. Pharm. Biomed. Anal., 2010, 52, 377-383.

42 S. Sakai, T. Ono, H. Ijima and K. Kawakami, Biomaterials, 2001, 22, 2827-2834.

43 S. Li, X. Jiao and H. Yang, Langmuir, 2013, 29, 1228-1237.

44 I. I. Slowing, J. L. Vivero-Escoto, C. W. Wu and V. S. Y. Lin, Adv. Drug Delivery Rev., 2008, 60, 1278-1288.

45 L. Palanikumar, E. S. Choi, J. Y. Cheon, S. H. Joo and J. H. Ryu, Adv. Funct. Mater., 2015, 25, 957-965.

46 T. Chen, W. Wu, H. Xiao, Y. Chen, M. Chen and J. Li, ACS Macro Lett., 2016, 5, 55-58.

47 S. Gadde, MedChemComm, 2015, 6, 1916-1929. 